\title{
Demixing and confinement of non-additive hard-sphere mixtures in slit pores
}

\author{
N. G. Almarza, C. Martín, E. Lomba, and C. Bores \\ Instituto de Química Física Rocasolano, CSIC, Serrano 119, E-28006 Madrid, Spain
}

(Received 16 October 2014; accepted 18 December 2014; published online 6 January 2015)

\begin{abstract}
Using Monte Carlo simulation, we study the influence of geometric confinement on demixing for a series of symmetric non-additive hard spheres mixtures confined in slit pores. We consider both a wide range of positive non-additivities and a series of pore widths, ranging from the pure two dimensional limit to a large pore width where results are close to the bulk three dimensional case. Critical parameters are extracted by means of finite size analysis. As a general trend, we find that for this particular case in which demixing is induced by volume effects, the critical demixing densities (and pressures) increase due to confinement between neutral walls, following the expected behavior for phase equilibria of systems confined by pure repulsive walls: i.e., confinement generally enhances miscibility. However, a non-monotonous dependence of the critical pressure and density with pore size is found for small non-additivities. In this latter case, it turns out that an otherwise stable bulk mixture can be unexpectedly forced to demix by simple geometric confinement when the pore width decreases down to approximately one and a half molecular diameters. (C) 2015 AIP Publishing LLC. [http://dx.doi.org/10.1063/1.4905273]
\end{abstract}

\section{INTRODUCTION}

Phase separation under confinement has been for decades a topic of primary interest both from the technological and fundamental science standpoints. ${ }^{1}$ It is obvious that the reduction in the number of neighbors of those molecules adjacent to the pore walls will induce important phase diagram shifts, whose character will be mostly dependent on the nature of the wall-fluid (or wall-adsorbate) interaction. In the limit of plain two dimensional confinement, the system will exhibit bidimensional criticality, which is essentially different-e.g., as critical indices are concerned-from its bulk three dimensional counterpart. ${ }^{2}$ We assume that this bidimensional criticality also holds for the different levels of confinement studied in this work. ${ }^{3-5}$ Nevertheless, in this work, the reliability of this assumption will be further checked for our particular case.

Many new and interesting effects can be induced by confining and the interplay between adsorbate-adsorbate and adsorbate-pore wall forces. Very recently, Severin and coworkers ${ }^{6}$ found evidence of a microphase separation in an otherwise fully miscible mixture of ethanol and water when adsorbed in a slit pore formed by a graphene layer deposited on a mica wall. Of utmost interest are also the effects that confinement has on enhancing or preempting crystallization of undercooled fluids. ${ }^{78}$ This has been a key approach in the attempts to throw some light in the search for the elusive liquid-liquid critical point in undercooled water, ${ }^{9}$ resorting to the preemption of crystallization induced by tight confinement of water in nanopores ${ }^{10,11}$ and extensive use of diffraction experiments in combination with computer simulations. In the field of colloidal science, numerous works have been devoted to the problem of demixing of colloid-polymer mixtures both in bulk and under confinement. In this latter instance, both symmetric mixtures adsorbed in slit pores with neutral walls ${ }^{4}$ and non-symmetric mixtures confined between attractive and repulsive walls $s^{5,12,13}$ have been studied. The effects of randomness and confinement on demixing of colloidpolymer systems have also been extensively investigated. ${ }^{14,15}$ Moreover, Fortini and Dijsktra ${ }^{16}$ explored the possibility of manipulating colloidal crystal structures by confinement in slit pores, stressing the potential technological implications of this class of studies. These colloid-polymer mixtures are mostly treated in terms of the Asakura-Oosawa model,,${ }^{17}$ by which the mixture is reduced to an effective one-component fluid and the demixing transition is in turn mapped onto a vapor-liquid transition.

On the other hand, explicit examples of two-component demixing have been studied in detail considering non-additive hard sphere (NAHS) mixtures. These are in fact some of the simplest statistical mechanical models that account for demixing induced by volume and/or entropic effects. Its limiting case, the Widom-Rowlinson (WR) model, ${ }^{18}$ has deserved particular theoretical attention and prompted the development of specially adapted algorithms to cope with the hard-core singularities and critical slowing down of the demixing transition. ${ }^{19}$ Góźdź has studied the demixing transition of the WR mixture under confinement by parallel neutral walls ${ }^{20}$ finding that purely geometric confinement favors mixing (i.e., increases the critical demixing density). More general instances of the non-additive hard sphere mixture problem (mostly in the symmetric case) have been studied in the two-dimensional limit ${ }^{21}$ and in a number of detailed studies in three dimensions. ${ }^{22-25}$ Duda and coworkers ${ }^{26}$ carried out a simulation and mean field study of the influence of confinement of the critical properties of the NAHS mixture and obtained results which turned out to be in qualitative agreement with those of Góźdź for the WR system: ${ }^{20}$ confinement by 
neutral walls in slit pores stabilizes mixing in a pure athermal system such as the NAHS mixture.

In this work, we intend to explore thoroughly the demixing transition of the symmetric non-additive hard sphere mixture under confinement in a slit pore by means of extensive computer simulation calculations and use of finite size scaling techniques to determine the critical properties. In contrast with previous works, our study includes a wider range of nonadditivities and pore widths. As a consequence, we will show, that despite the general trend found in previous works, ${ }^{4,20,26}$ there is a range of pore widths close to the bi-dimensional limit for which systems with small non-additivities exhibit a nonmonotonous dependence of the critical density and pressure on the pore width. To the best of our knowledge, this behavior has not been described or accounted for up to the present.

Explicitly, our model is defined as a mixture of A and B components, characterized by an interaction of the type

$$
u_{\alpha \beta}(r)=\left\{\begin{array}{ll}
\infty & \text { if } r \leq \sigma\left(1+\left(1-\delta_{\alpha \beta}\right) \Delta\right) \\
0 & \text { if } r>\sigma\left(1+\left(1-\delta_{\alpha \beta}\right) \Delta\right)
\end{array},\right.
$$

where $\alpha, \beta$ denote the A and B species, $\delta_{\alpha \beta}$ is Kronecker's delta, the non-additivity parameter is $\Delta>0$, and $r$ is the interparticle separation.

We will here study a series of confined non-additive hard sphere mixtures (for various $\Delta>0$ values) using extensive semi-grand ensemble Monte Carlo (MC) simulations. ${ }^{23,27,28}$ The effects of geometric confinement are modeled by the presence of hard-core (i.e., neutral) walls, separated by a distance, $H$, that constrain the particle movement in one space direction (along the $z$-axis as defined here). The fluid particle would thus be subject to an external potential of the form

$$
V^{\text {ext }}(z)=\left\{\begin{array}{ll}
0 & \text { if } \sigma / 2 \leq z \leq H-\sigma / 2 \\
\infty & \text { otherwise }
\end{array} .\right.
$$

This aims at reproducing the behavior of a fluid confined in a slit pore. Since all interactions at play are purely hard-core, the demixing transition will result from the interplay of entropic and enthalpic (i.e., excluded volume) effects. Our calculations range from the pure two dimensional limit to a relatively large pore width $(10 \sigma$, approaching the bulk three dimensional mixture). We have taken advantage of the particular nature of the interaction to implement a cluster algorithm ${ }^{25,29,30}$ in order to cope with the critical slowing down when approaching the consolute point. Finite size scaling techniques have been applied in order to provide accurate estimates of the critical points. ${ }^{23}$ As mentioned, this class of systems was previously studied by Duda $e t$ al. ${ }^{26}$ by means of mean-field theory and Monte Carlo simulations, considering two values of the slit width, $H$, away from the two dimensional limit, and various non-additivities, $\Delta$. In most of the cases, these authors simulate just one system size, corresponding to a number of particles $N=1000$. Here, we will perform a comprehensive analysis of the phase diagram for a wider range of values of $H$ (starting from $H=\sigma$ ) and $\Delta$. This will uncover the non-monotonous dependence of the critical behavior on the confinement. In addition, for each case, several values of $N$ will be considered, which will allow us to get more reliable estimates of the phase diagram of these systems, and in particular of the critical points.

The rest of the paper is sketched as follows: in Sec. II, we briefly summarize the computer simulation techniques we have used, and our main results are presented together with conclusions and future prospects in Sec. III.

\section{METHODOLOGY}

Given the particular symmetry of our model, the most appropriate simulation approach to study the phase equilibria is the use of semi-grand canonical MC simulations. ${ }^{23,27,28} \mathrm{We}$ impose the difference between the chemical potentials of the two components $\Delta \mu \equiv \mu_{B}-\mu_{A}$, the volume $V$, the temperature $T$, and keep the total number of particles, $N\left(=N_{\mathrm{A}}+N_{\mathrm{B}}\right)$ fixed; $x=N_{\mathrm{A}} / N$ is the concentration of particle species A. The total number density $\rho=N / V$ is thus fixed. In addition to the conventional MC moves, particles can also modify their identity (i.e., the species to which they belong). ${ }^{22}$ The identity sampling can be performed through an efficient cluster algorithm that involves all the particles in the systems and that will be presented later in the paper. After $5 \times 10^{5} \mathrm{MC}$ sweeps for equilibration, our simulations were typically extended over $2 \times 10^{6} \mathrm{MC}$ sweeps to perform averages. A sweep involves $N$ single-particle translation attempts and one cluster move. Note that due to symmetry, the critical mole fraction of component $A$ (and $B$ ) will be $x_{c}=1 / 2$, and the demixing transition will occur at $\Delta \mu=0$. When demixing occurs, the mole fraction, $X$ of the components in the two phases, is computed through an ensemble average considering the order parameter

$$
\theta=2 x-1,
$$

as $X=1 / 2 \pm \sqrt{\left\langle\theta^{2}\right\rangle} / 2$. Given the symmetry of the model and the efficiency of the cluster algorithm, the average of $x$ from the simulations at $\Delta \mu=0$ will be $\langle x\rangle \simeq 1 / 2$, independently of the presence or absence of demixing at the simulation conditions. By analysis of the mole fraction histograms for a series of binary mixtures at different total densities, $\rho=\rho_{A}+\rho_{B}$, one can obtain a series of phase diagrams for each sample size, as illustrated in Figure 1, where the extreme size dependence of the results on the sample size in the neighborhood of the critical point can be readily appreciated.

It is well known that as the critical point is approached, larger samples are needed, correlations become long ranged, and critical slowing down must be dealt with somehow. To that aim, we have complemented single particle moves with cluster moves ${ }^{25,29,30}$ following the Swendsen-Wang strategy. ${ }^{29}$ Two particles of the same species are considered linked within the same cluster when their separation is less than $\sigma(1+\Delta)$. Note that due to the linking criteria and the hard-core interactions, all the particles belonging to a given cluster are of the same species, and two particles lying at a distance below $\sigma(1+\Delta)$ are necessarily included in the same cluster. As a consequence, cluster identity swaps do not lead to particle overlaps, and for the symmetric case, $\Delta \mu=0$, the procedure leads to a rejectionfree algorithm of composition sampling for a fixed set of particle positions. This algorithm rests on two key elements: (1) clusters are built following the rules defined above, and 


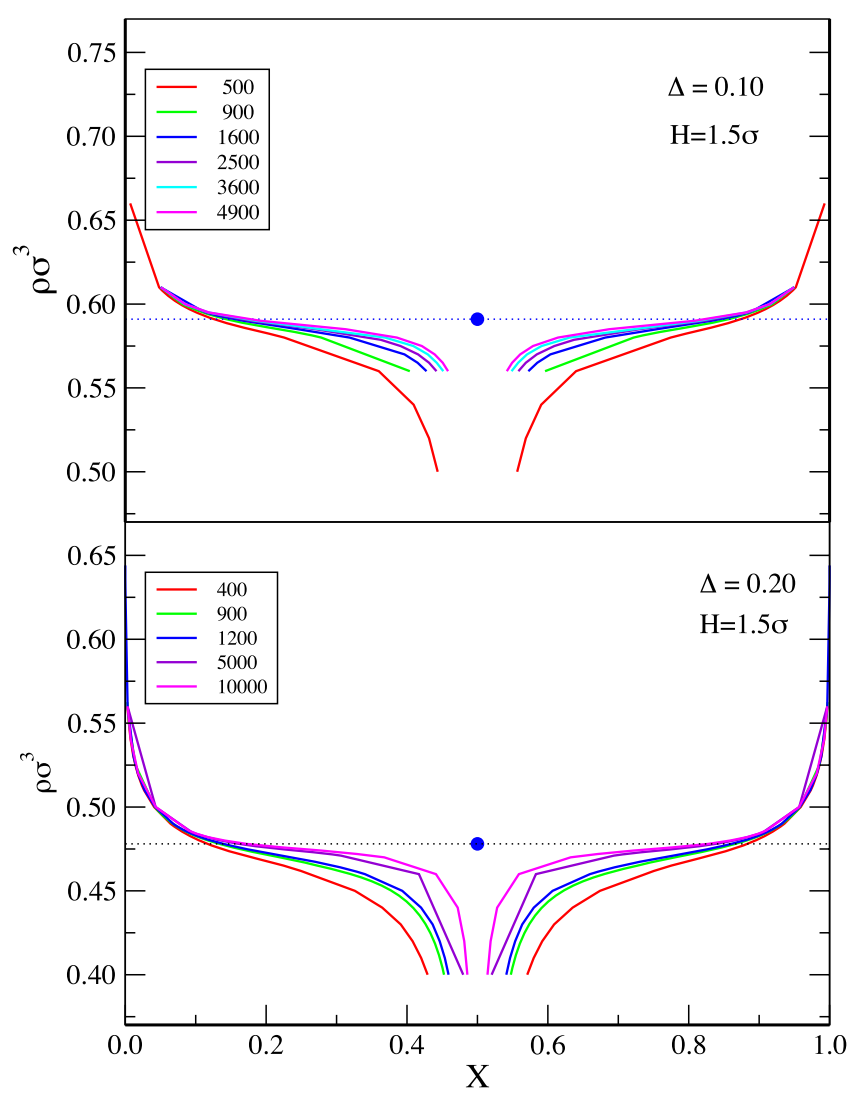

FIG. 1. Size dependence of phase diagram of symmetric non-additive mixtures (with $\Delta=0.1$ and $\Delta=0.2$ ) confined in a slit pore of width $H=1.5 \sigma$. The dotted line marks the estimate for the critical density as obtained from the finite-size scaling analysis. The symbol corresponds to the critical point in the density-mole fraction plane.

(2) for each cluster, one of the two possible identities ( $A$ or $B)$ is independently chosen with equal probabilities. Notice that, for a given non-overlapping configuration of the system, the partition of the particles into clusters is deterministic, in the sense that it does not depend on the current identity of the different clusters. As a consequence, for the symmetric case, $\Delta \mu=0$, the probabilities of belonging to species $A$ or to species $B$ are the same for each individual cluster, and each of the equally probable $2^{N_{c}}$ realizations of the overall cluster labeling has the same probability of being generated with the sampling procedure ${ }^{19}\left(N_{c}\right.$ being the number of clusters for a given set of position coordinates). Along the simulations, the fraction of configurations containing percolating clusters is monitored as an additional signal of the presence of a phase transition. $^{31}$

Another issue that has to be addressed is the calculation of the pressure in the confined system with discontinuous interactions. The scheme proposed by de Miguel and Jackson ${ }^{32}$ and further exploited for the Widom-Rowlinson mixture in Ref. 33 turns out to be the simplest approach in the present case. In order to estimate the pressure, we perform virtual compressions of the system (both in the $z$ directionorthogonal to the pore walls-and in the $x, y$ directions). The virial pressure is then computed as

$$
P^{z, x y} / k_{B} T=\lim _{\Delta V_{z, x y} \rightarrow 0}\left\langle\rho+\frac{1}{\Delta V_{z, x y}} n_{o}(\Delta V)\right\rangle,
$$

where $k_{B}$ is Boltzmann's constant and $T$ the absolute temperature as usual, $\Delta V$ is the change of the volume in the compression, $\Delta V=V-V^{\text {test }}$, with $\Delta V>0$, and $n_{o}(\Delta V)$ is the number of particle pairs that overlap during the virtual (test) compression of the system. In practice, the pressure is calculated by computing $n_{o}$ for a set of values of $\Delta V$ and extrapolating to the limit $\Delta V \rightarrow 0$. Note that in an inhomogeneous system such as this one, there is no straightforward generalization of the virial pressure expression of bulk fluids. More precisely, in narrow slits, mechanical equilibrium is no longer correlated with a bulk pressure, but with the normal component of the pressure tensor with respect to the walls, and this quantity is connected with the value of the density profile at contact with the walls via the corresponding statistical mechanical sum rule. ${ }^{34}$ The method adopted here is more appropriate in the context of computer simulation.

Now, the demixing transition is monitored following the evolution and size dependence of a series of appropriate order parameters. Here, we have considered on one hand, $\theta$, as defined in Eq. (3), and on the other, the fraction of percolating configurations, $\chi$. A configuration is defined as percolating if (and only if) at least one of its clusters becomes of infinite size when considering the periodic boundary conditions; those clusters are often denoted as wrapping clusters. With the $\theta$ order parameter, we proceed to perform a Binder cumulant like analysis. ${ }^{35,36}$ This is done by considering ratios between momenta of the order parameter probability distribution given as

$$
U_{2 n}=\frac{\left\langle\theta^{2 n}\right\rangle}{\left\langle\theta^{2}\right\rangle^{n}},
$$

where the angular brackets indicate ensemble averages, and looking at how these quantities vary with the density for different system sizes. Calculations are carried out for different sample sizes, $N$, and curves of $\chi, U_{4}$, and $U_{6}$ are plotted vs. total density $\rho$. According to the finite size scaling analysis, ${ }^{36}$ the crossing of the curves $U_{2 n}(\rho)$ for different system sizes should define the critical point and be size independent for sufficiently large samples. In practice, we fit the critical density estimates, $\rho_{c}(N)$, obtained from different crossings. This is done by taking pairs of system sizes, $N_{i}<N_{j}$, and looking for the density $\rho_{c}\left(N_{j} \mid N_{i}\right)$, where the curves of the analyzed property for the two system sizes cross. The results $\rho_{c}\left(N_{j} \mid N_{i}\right)$ for a given $N_{i}$ are taken as estimates for the pseudo-critical densities for the system size $N_{j}$, and from then, one can extrapolate the critical density in the thermodynamic limit $\left(1 / N_{j} \rightarrow 0\right)$. These extrapolations were done by fitting the results to straight lines of the form ${ }^{23} \rho_{c}(N)=\rho_{c}+a N^{-1 /(2 v)}$, where we took $v=1$, according to the assumed bidimensional criticality. Notice that a more rigorous finite-size scaling analysis should be based on results from simulations carried out in either $(N, p, T, \Delta \mu)$ or $\left(\mu_{A}, V, T, \Delta \mu\right)$ ensembles instead of resorting to $(N, V, T$, $\Delta \mu)$ semi-grand ensemble simulations. ${ }^{37-39}$ However, different studies found in the literature ${ }^{20,23,40}$ have shown that for NAHS systems, one can rely on semi-grand ensemble simulations at constant volume and number of particles. For practical reasons, the use of this ensemble is also profitable since, for instance, the difficulties in the sampling of the number of particles in the grand canonical ensemble for systems 


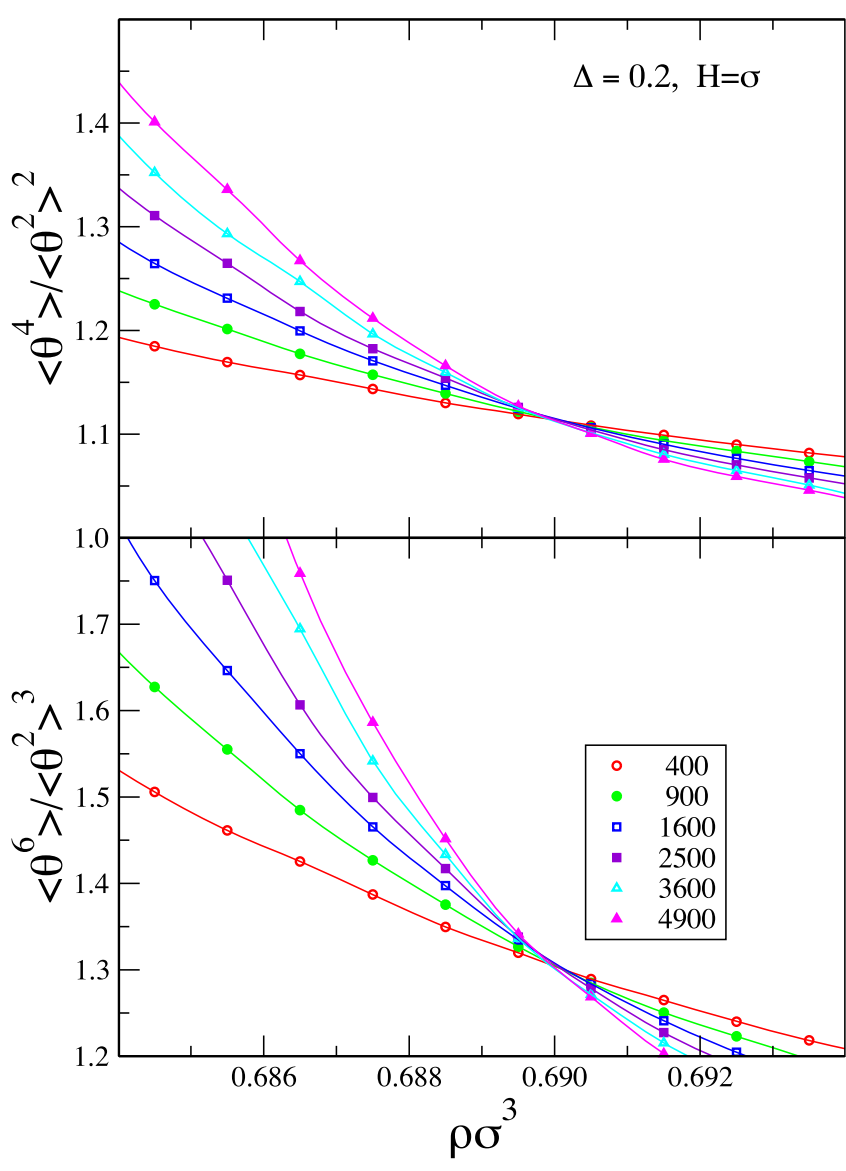

FIG. 2. Size dependence of the $U_{4}$ and $U_{6}$ cumulants of the order parameter $\theta$ for the symmetric non-additive hard sphere mixture.

with high critical densities are avoided. The estimates of $\rho_{c}$ obtained from the fraction of percolating configurations and the cumulants are fully consistent within statistical error bars. The corresponding critical pressures are obtained by means of a series of semi-grand canonical simulations carried out at the critical density and $\Delta \mu=0$ with varying sample sizes and extrapolating $P^{x y} / k_{B} T$ and $P^{z} / k_{B} T$ for $1 / N \longrightarrow 0$. An example of the evolution of the order parameters for the two dimensional limit (i.e., pore width $H=\sigma$ ) and non-additivity $\Delta=0.2$ is presented in Figures 2 and 3 .

For densities about $\rho_{c}$, demixing occurs at $\Delta \mu=0$. The mole fractions of the coexisting phases for each given system size are computed through the order parameter $\theta$ as $X_{ \pm}=\frac{1}{2}\left[1 \pm \sqrt{\left\langle\theta^{2}\right\rangle}\right]$. Using the results for different system sizes, we estimate the composition in the thermodynamic limit by fitting the results to a second-order polynomial in $(1 / N)$. Then, the $X-\rho$ phase diagram can be fully estimated discarding the equilibrium data close to the critical $\rho_{c}$ (much affected by sample size dependence), and using the extrapolated data $X(\rho)$, and a fit to the approximate ${ }^{37}$ scaling law

$$
\left|\frac{X(\rho)-x_{c}}{x_{c}}\right| \propto\left|\frac{\rho}{\rho_{c}}-1\right|^{\beta},
$$

where we assume the system to belong to the two dimensional Ising universality class, ${ }^{23}$ and hence, $\beta=1 / 8$. On theoretical grounds, ${ }^{3}$ one expects that sufficiently close to the critical point a fluid confined in a slit pore should strictly comply with

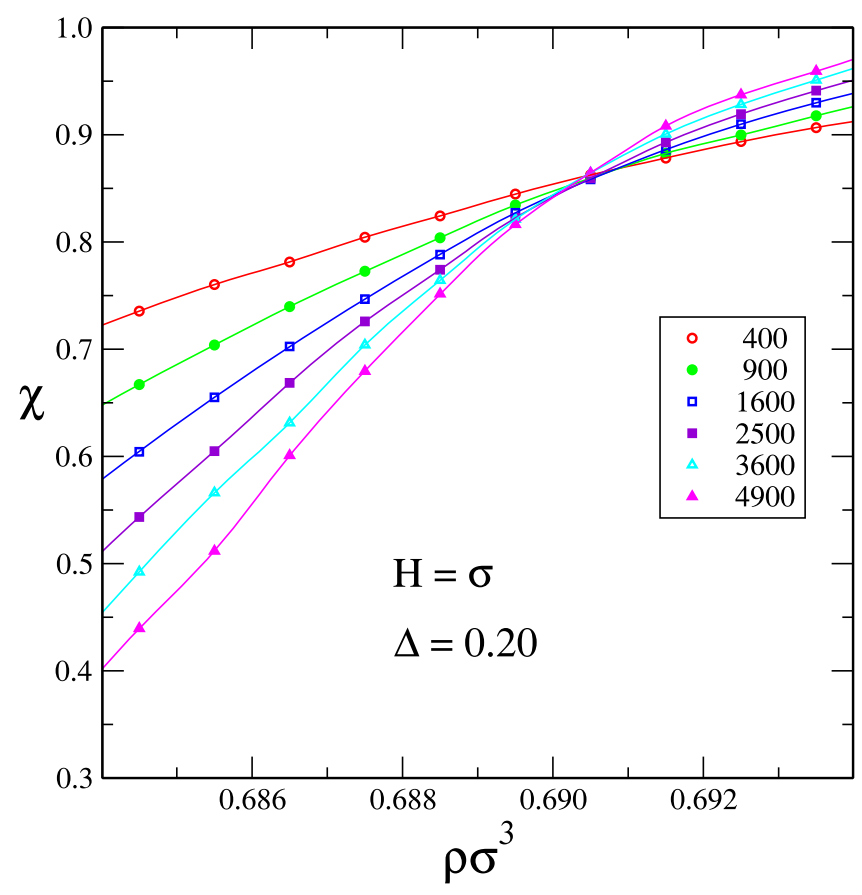

FIG. 3. Size dependence of the fraction of percolating configurations, $\chi$, for the symmetric non-additive hard sphere mixture.

a two dimensional criticality. Nonetheless, in Refs. 4 and 5, a rigorous analysis of the crossover between two and three dimensional critical behavior was carried out, and "effective" critical indices (dependent on the pore width) were determined. In these works, it was found that these effective critical exponents lack a fundamental meaning, since they depend on the range of temperatures (or densities in our case) that are used for the analysis. ${ }^{5}$ Moreover, according to the results of Rouault et al. ${ }^{4}$ one should not expect significant departures from the two-dimensional scaling for pore widths below $H=10 \sigma$. Consequently, for practical purposes, since we are dealing with relatively narrow pores (or equivalently thin films), the plain two dimensional Ising scaling relations are a good approximation. ${ }^{5}$ In addition to the previous discussion, we present numerical evidence in Sec. III of the appropriateness of the assumption of two dimensional criticality.

\section{RESULTS}

Before presenting the results for different cases of $\Delta$ and $H$, we will address the issue of the type of criticality of systems confined in slit pores. To this aim, we have selected a rather wide pore $H=10$, and a value $\Delta=0.2$. The analysis of the type of criticality has been carried from the results of simulations in the grand canonical ensemble $(\mu, \Delta \mu, V, T)$, for different values of the lateral length of the system $L=\sqrt{V / H}$, considering $\Delta \mu=0$. From finite-size scaling arguments, ${ }^{36}$ if the criticality of the demixing transition belongs to the two-dimensional Ising universality class, the crossing of the $U_{4}(\mu)$ curves for different system sizes, $L$, is expected to occur close to the critical value $\mu_{c}$, and at value ${ }^{41} U_{4}^{c} \simeq 1.168$ : the results shown in Fig. 4 (left) are fully compatible with that behavior. On the other hand, the scaling behavior of the $U_{4}$ around the critical 


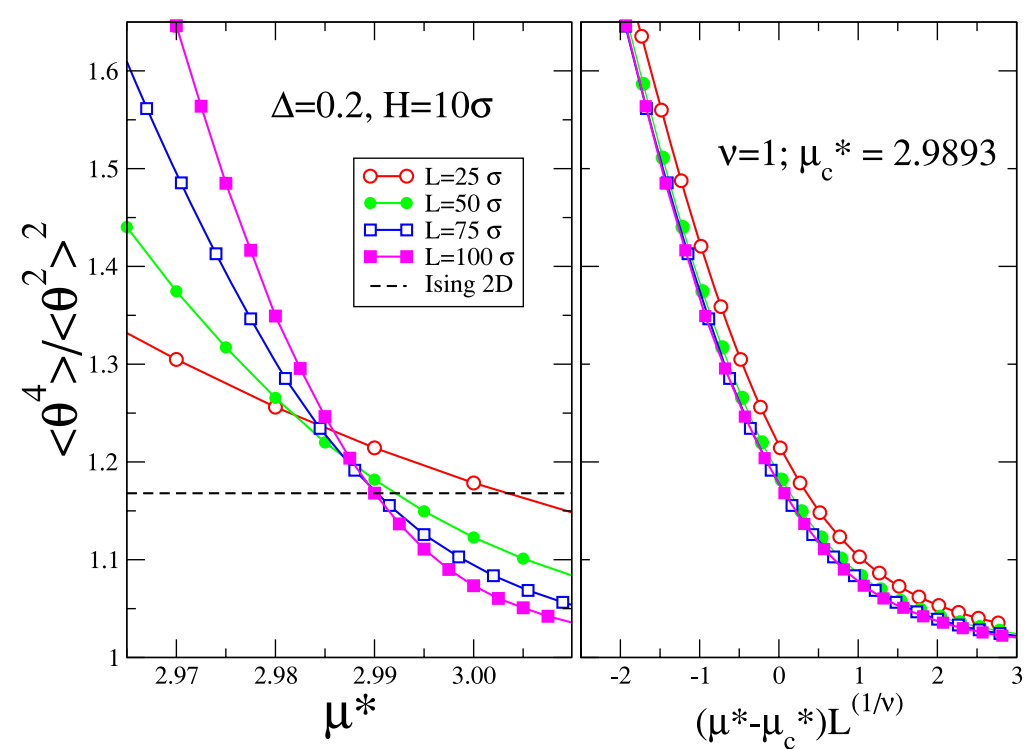

FIG. 4. Scaling behavior of the Binder cumulant, $U_{4}$ as a function of the reduced chemical potential, $\mu^{*}$ $=\mu / k_{B} T$, for systems with $\Delta=0.2, H=10$, and different values of $L$. On the left panel, it can be seen how the crossings between different curves approach the universal value $U_{4}^{c} \simeq 1.168$ of the two-dimensional Ising universality class (indicated with a dashed line) for the largest system sizes. On the right panel, it tests the scaling behavior of $U_{4}(L)$ around the critical point against that expected for 2D criticality $(v=1)$ : notice that only the results for $L=25 \sigma$ deviate from the expected common line (the estimate for the critical point was $\mu_{c}^{*} \simeq 2.9893$ ). point is shown in Fig. 4 (right panel). The results for system sizes $L \geq 50 \sigma$ collapse in a single curve when represented as a function of the scaled variable $L^{1 / v}\left(\mu-\mu_{c}\right)$, when considering the value $v=1$ of the two-dimensional Ising criticality. Since, in the systems considered in this work, the largest pore width is precisely $H=10$, we are fully confident in the analysis of the critical point under the assumption of two-dimensional Ising criticality.

We have considered systems with varying degrees of nonadditivity, ranging from $\Delta=0.1$ to $\Delta=1$, and pore widths from $\sigma$ to $10 \sigma$ (see Table I for the specific values).

Semi-grand ensemble simulations were run for samples of 400,900,1600,2500, 3600, and 4900 particles when $H<5.5 \sigma$. Sample sizes of 6400 particles were included for pore widths larger than $5.5 \sigma$ up to $H=10 \sigma$, where an additional sample size of 8100 particles was included. As mentioned in Sec. II, for a given system defined by a pair $(H$, $\Delta)$, simulations are run for a series of total densities, $\rho$, and we monitored the behavior of the order parameters (as illustrated in Figures 2 and 3). Following the procedures indicated above, we obtain a series of phase diagrams as illustrated in graphs of Figure 5 for three selected pore widths, $H=\sigma, 2.5 \sigma$, and $10 \sigma$. The complete set of critical properties for most of the systems studied is collected in Table I.

From Figure 5, one immediately appreciates that increasing the non-additivity lowers the critical density, i.e., favors demixing as expected. In contrast, we observe that confinement tends to stabilize the mixed phase. This effect is particularly visible when going from the $H=2.5 \sigma$ system to the two dimensional case, where one sees that the critical density practically doubles for the two largest non-additivities. Obviously, as the non-additivity decreases demixing occurs at higher

TABLE I. Critical parameters for non-additive hard sphere mixtures confined in slit pores. Error estimates of critical densities and pressures are below the last significant digits in both instances. Here, $P^{*}=P \sigma^{3} / k_{B} T$.

\begin{tabular}{|c|c|c|c|c|c|c|c|c|c|c|c|c|}
\hline \multirow[b]{2}{*}{$H / \sigma$} & \multicolumn{3}{|c|}{$\Delta=0.1$} & \multicolumn{3}{|c|}{$\Delta=0.2$} & \multicolumn{3}{|c|}{$\Delta=0.5$} & \multicolumn{3}{|c|}{$\Delta=1$} \\
\hline & $\rho_{c} \sigma^{3}$ & $P_{c}^{x y *}$ & $P_{c}^{z *}$ & $\rho_{c} \sigma^{3}$ & $P_{c}^{x y *}$ & $P_{c}^{z *}$ & $\rho_{c} \sigma^{3}$ & $P_{c}^{x y *}$ & $P_{c}^{z *}$ & $\rho_{c} \sigma^{3}$ & $P_{c}^{x y *}$ & $P_{c}^{z^{*}}$ \\
\hline 1.00 & 0.841 & 8.30 & $\ldots$ & 0.690 & 3.84 & $\ldots$ & 0.460 & 1.314 & $\ldots$ & 0.286 & 0.547 & $\ldots$ \\
\hline 1.05 & 0.802 & 7.91 & 16.95 & 0.657 & 3.66 & 13.85 & $\ldots$ & $\ldots$ & $\ldots$ & $\ldots$ & $\ldots$ & $\ldots$ \\
\hline 1.10 & 0.765 & 7.55 & 8.67 & 0.628 & 3.49 & 7.01 & 0.419 & 1.193 & 4.64 & $\ldots$ & $\ldots$ & $\ldots$ \\
\hline 1.25 & 0.679 & 6.64 & 4.08 & 0.555 & 3.07 & 3.05 & 0.370 & 1.051 & 1.919 & $\ldots$ & $\ldots$ & $\ldots$ \\
\hline 1.50 & 0.591 & 5.52 & 3.86 & 0.477 & 2.56 & 2.10 & 0.314 & 0.876 & 1.089 & 0.194 & 0.365 & 0.621 \\
\hline 1.75 & 0.607 & 4.70 & 7.91 & 0.445 & 2.18 & 2.54 & 0.280 & 0.751 & 0.912 & $\ldots$ & $\ldots$ & $\ldots$ \\
\hline 2.00 & 0.699 & 4.71 & 8.31 & 0.477 & 1.97 & 3.62 & 0.266 & 0.660 & 0.959 & 0.155 & 0.275 & 0.405 \\
\hline 2.25 & 0.675 & 4.97 & 4.83 & 0.488 & 1.97 & 2.75 & 0.262 & 0.604 & 0.889 & $\ldots$ & $\ldots$ & $\ldots$ \\
\hline 2.50 & 0.638 & 4.69 & 4.41 & 0.476 & 1.97 & 2.25 & 0.257 & 0.575 & 0.795 & 0.138 & 0.227 & 0.319 \\
\hline 2.75 & 0.635 & 4.24 & 5.44 & 0.461 & 1.88 & 2.16 & 0.252 & 0.558 & 0.735 & $\ldots$ & $\ldots$ & $\ldots$ \\
\hline 3.00 & 0.656 & 4.16 & 5.54 & 0.456 & 1.78 & 2.21 & 0.247 & 0.542 & 0.693 & 0.128 & 0.202 & 0.268 \\
\hline 3.50 & 0.641 & 4.20 & 4.40 & 0.456 & 1.73 & 2.04 & 0.239 & 0.512 & 0.632 & 0.122 & 0.189 & 0.241 \\
\hline 4.00 & 0.642 & 3.97 & 4.66 & 0.449 & 1.67 & 1.93 & 0.233 & 0.491 & 0.592 & 0.117 & 0.180 & 0.221 \\
\hline 4.50 & 0.638 & 3.96 & 4.27 & 0.446 & 1.63 & 1.86 & 0.229 & 0.478 & 0.562 & 0.113 & 0.173 & 0.207 \\
\hline 5.00 & 0.636 & 3.85 & 4.29 & 0.443 & 1.61 & 1.80 & 0.225 & 0.468 & 0.541 & 0.109 & 0.167 & 0.196 \\
\hline 6.00 & 0.634 & 3.78 & 4.11 & 0.439 & 1.57 & 1.73 & 0.220 & 0.452 & 0.508 & 0.105 & 0.160 & 0.182 \\
\hline 7.50 & 0.631 & 3.72 & 3.94 & 0.436 & 1.54 & 1.66 & 0.215 & 0.440 & 0.482 & 0.100 & 0.153 & 0.169 \\
\hline 10.00 & 0.630 & 3.65 & 3.82 & 0.432 & 1.51 & 1.59 & 0.210 & 0.428 & 0.458 & 0.096 & 0.147 & 0.158 \\
\hline
\end{tabular}




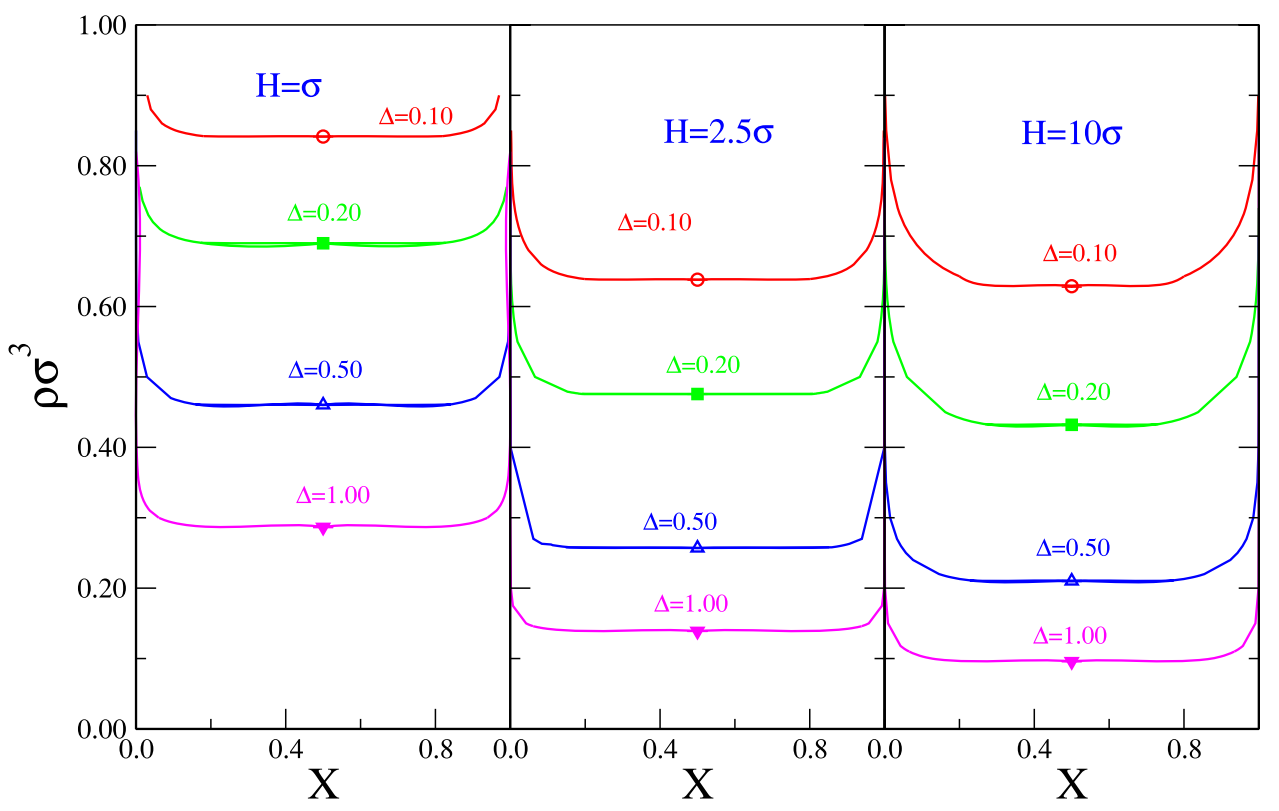

FIG. 5. Phase diagram of the non-additive hard-sphere mixtures for various pore widths and non-additivity parameters.

packing fractions and packing constraints necessarily limit the effects of confinement on the critical density. Interestingly, we observe that as $H>2.5 \sigma$, the change on the critical density is much smaller and practically negligible for the smallest non-additivity. In practice, as we will see later, for $H=10 \sigma$, the critical values of the bulk three dimensional hard sphere mixture have almost been reproduced. This effect of stabilization of the mixture due to confinement can be easily understood when one realizes that the average number of neighbors is reduced as one goes from the bulk three dimensional system to the two dimensional one. This implies that particles of a given type A (or B) will have fewer neighbors of type B (or A) when they are close to the walls, the limiting case being the two dimensional system. As a consequence, these particles will have a lower tendency to demix as the density (or pressure) is increased. Obviously, the fraction of particles adjacent to the walls is maximum when $H=\sigma$, and this fraction decreases rapidly as $H$ increases, and as a consequence the critical density decreases. Once the pore allows for two fluid layers inside, the fall in the critical density as the pore widens is not so pronounced.

Now, in Figures 6 and 7, we observe the explicit evolution of the critical density vs $1 / H$ and the critical pressure vs $H$. In Figure 6, some values from the literature for the two and three dimensional limit are included. As mentioned before, the critical densities for $H=10 \sigma$ practically have already converged to those of the unconfined system. The dependence of the critical density on pore size has two linear regimes, which for $\Delta=1$ and $1 / 2$ merge continuously at $H \approx 1.5 \sigma$. The linear dependence of the critical density on the inverse of the pore width can be explained in terms of the scaling relations ${ }^{4,42}$ which actually determine

$$
\rho_{c}(H)-\rho_{c}(H=\infty) \propto 1 / H^{1 / v},
$$

where $v$ is the correlation length critical exponent, which happens to be $v=1$ for 2D Ising universality class and $v=0.630$ in the $3 \mathrm{D}$ case. ${ }^{4}$ From the figure is rather evident that our system essentially follows a two dimensional criticality, as argued before. Moreover, close to the two dimensional limit, the change in the critical density with the pore width can be explained simply in terms of the increase of available volume, keeping constant the surface density corresponding to the limit $H=\sigma$, i.e., we can write ${ }^{13}$

$$
\rho_{c}(H) \approx \frac{\rho_{c}(H=\sigma)}{H}
$$

In Figure 6, this equation is represented by dashed-dotted lines, and in fact, one observes that the dependence of $\rho_{c}(H)$ is well captured up to $H=1.5 \sigma$. The quasi-two dimensional character of the transition in this limiting regime is further stressed in the lower panel of Figure 6, where the critical densities are scaled with the cross-interaction diameter squared, $\sigma_{A B}^{2}$, and one clearly sees that for $\Delta \leq 0.5$, the curves practically collapse when approaching $H=\sigma$. This simply implies that the transition is controlled by the excluded surface between $\mathrm{A}$ and $\mathrm{B}$ particles.

On the other hand, one might assess to what extent Kelvin's equation, ${ }^{43}$ which also predicts a $1 / H$ dependence for $\rho_{c}$, applies to our problem. For the particular case of a mixture, one should expect that the change of the chemical potential of the adsorbed fluid with the respect to the value at the binodal (at saturation in terms of vapor-liquid equilibrium) should be ${ }^{13}$

$$
\Delta \mu \propto \frac{\gamma_{w A}-\gamma_{w B}}{H},
$$

where $\gamma_{w A(B)}$ is the surface tension between component $\mathrm{A}(\mathrm{B})$ and the wall. Clearly our symmetric mixture leads to $\Delta \mu=0$, by which one should not expect any change in the critical demixing density due to confinement. And in fact, if one considers the behavior as $1 / H \longrightarrow 0$, for small non-additivities, certainly the slope of $\rho_{c}(H)$ is close to zero. One can actually go a bit beyond that using the mean-field approach of Duda 


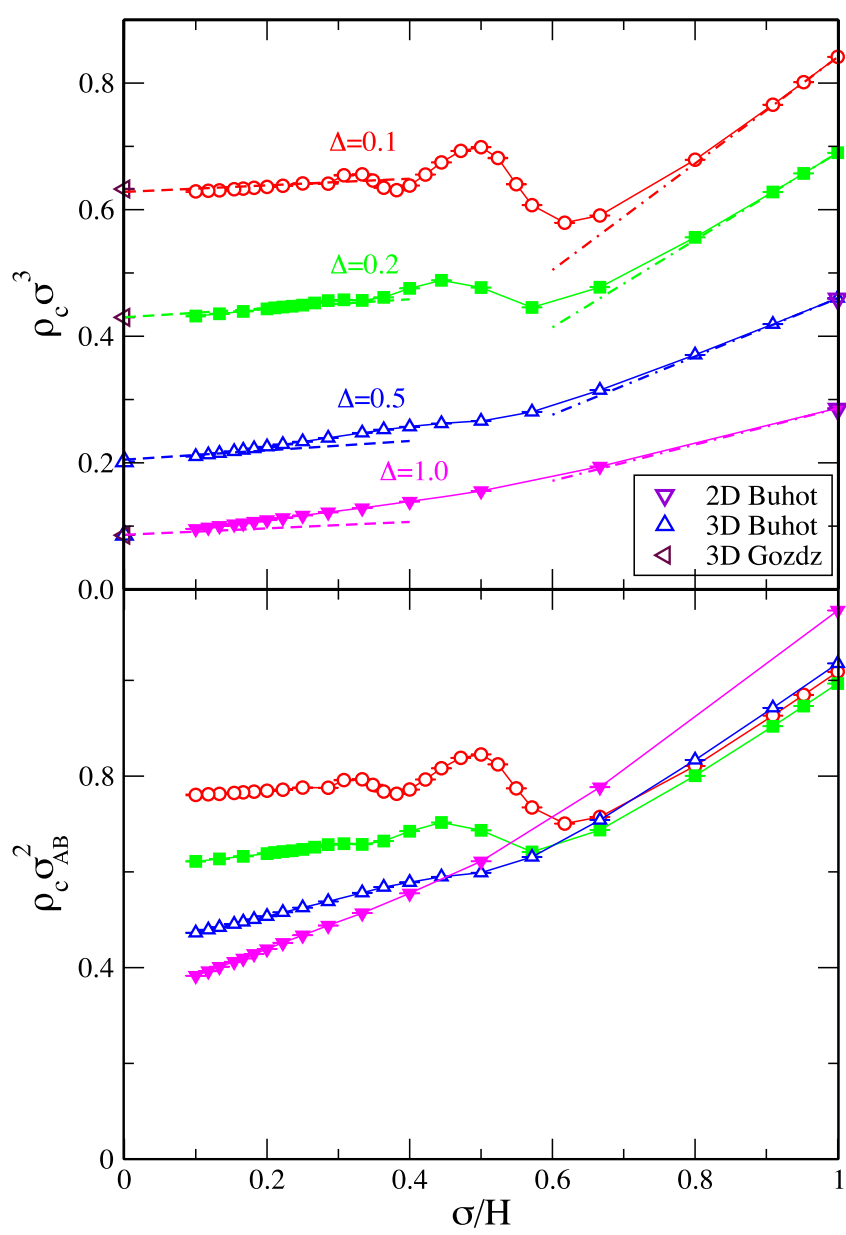

FIG. 6. Critical density dependence on the slit pore size as computed in this work and compared with limiting values in $2 \mathrm{D}$ and $3 \mathrm{D}$ by $\mathrm{Buhot}^{25}$ and by Góźdź ${ }^{23}$. Dotted-dashed lines represent the quasi-two dimensional limiting behavior described by Eq. (8), and dashed ones, linear contribution (10) when $1 / H \rightarrow 0$ - of the mean field approximation. ${ }^{26}$ In the lower panel, the same data are represented scaled with the cross-interaction diameter squared (see discussion in the text).

et al. ${ }^{26}$ which for small $1 / H$ predicts

$$
\rho_{c}(H)=\rho_{c}(H=\infty)\left(1+\frac{2(6+3 \Delta)}{w^{\prime}\left(\pi \rho_{c}(H=\infty) \sigma^{3} / 6\right)} \frac{\sigma}{H}\right)
$$

with

$$
w^{\prime}(x)=\frac{12\left(x^{2}-2(1+x)\right)}{(1-x)^{4}} .
$$

Equation (10) is represented in Figure 6 by dashed lines, and one clearly observes that the linear dependence of the $\rho_{c}(H)$ curves when approaching the three dimensional limit is well captured, with the exception of the largest non-additivity, $\Delta=1$, which departs somewhat from the mean field prediction.

The overall picture can be qualitatively explained when one simply realizes that for smaller pore sizes, the critical densities grow rapidly as the pore shrinks due to the marked decrease in the number of neighbors induced by the presence of walls. On the other hand, between the two linear regimes described above, an interesting feature emerges in the region $1.5 \sigma<H<4 \sigma$ for $\Delta=0.1$ and 0.2 : both the critical density and $P_{c}^{z} / k_{B} T$ show a clear non-monotonous dependence on $\mathrm{H}$, with maxima located at $H \approx \sigma, 2 \sigma, 3 \sigma$, and $4 \sigma$, 一the latter

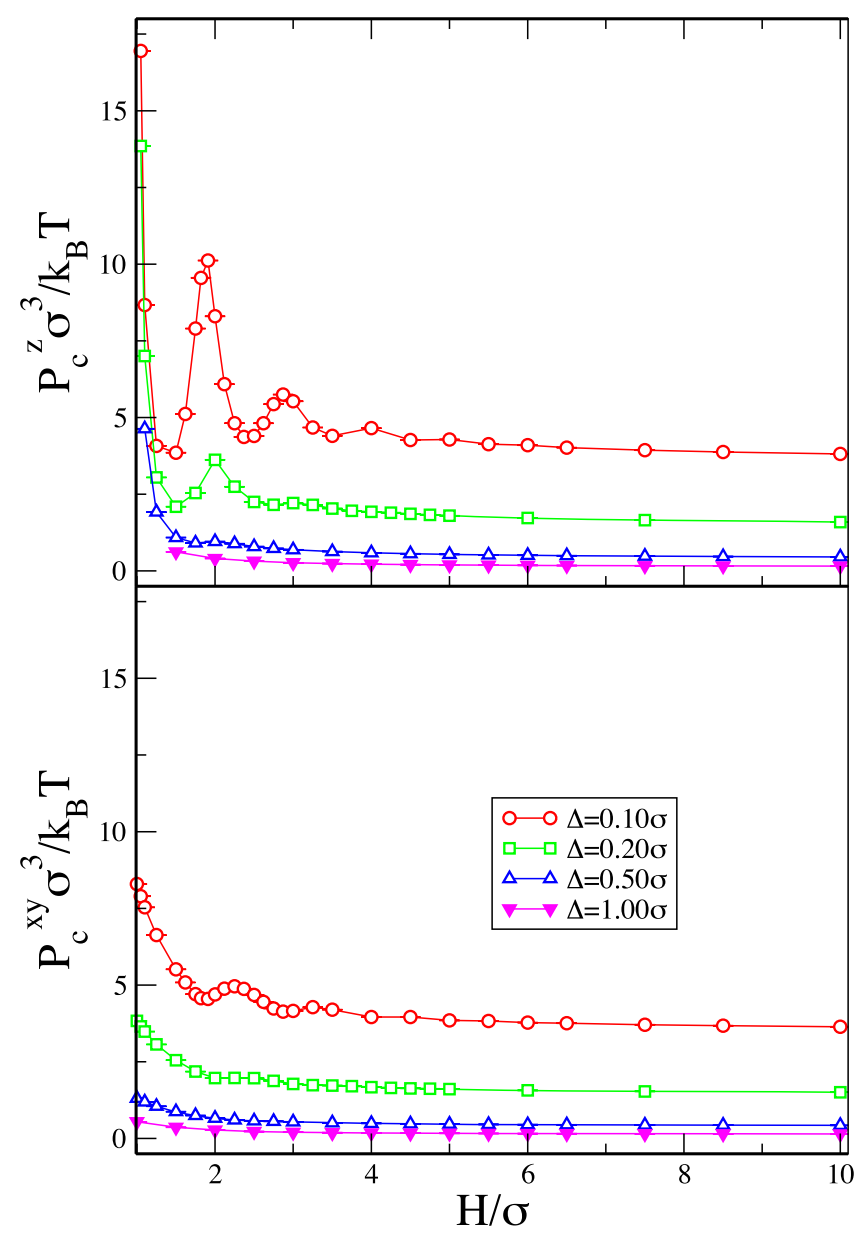

FIG. 7. Critical pressure dependence on the slit pore size for various nonadditivity parameters. Upper graph corresponds to the pressure on the pore walls and the lower graph to the corresponding transverse components.

only visible in the pressure curve-recalling the neighbor shell structure of a pair distribution function. We find then that in the ranges $(n-1 / 2) \sigma \lesssim H \lesssim n \sigma(n=2,3)$, the critical density and pressure increase (i.e., the mixture is stabilized) when the pore widens. Note however that on $P_{c}^{x y} / k_{B} T$, the maxima are shifted towards larger $H$-values, and actually, the minima of $P_{c}^{x y} / k_{B} T$ lie close to the maxima of $P_{c}^{z} / k_{B} T$. Somehow, the increase in the pressure against the pore walls tends to be compensated by a decrease of the pressure along the unbound directions. This mismatch is the obvious result of the lack of isotropy induced by the walls.

An extreme situation occurs at $H=1.5 \sigma$ and $\Delta=0.1$ for which the critical density $\left(\rho_{c} \sigma^{3}=0.591\right)$ is appreciably lower than that of the bulk ${ }^{23}\left(\rho_{c} \sigma^{3}=0.6325(8)\right)$. This actually implies that for certain systems (i.e., degrees of non-additivity), a stable mixture can be forced to demix by simple geometric confinement. In fact, one observes that the maxima in $\rho_{c}$ i.e., local stability maxima for the mixtures-occur when the pore can fit approximately an integer number of layers $(1,2$, and 3). From these state points, increasing or decreasing the pore size induces demixing. The effect of the increase in pore size is easily explained as the result of an increasing number of neighbors of different species that will prefer to be in a single component phase. On the other hand, if we focus on the behavior of the system when going from $H \approx 2 \sigma$ to $H \approx 1.5 \sigma$, 
we realize that the number of neighbors does not dramatically change when $H$ varies within these limits, as long as $\Delta$ is small. In fact, for $\Delta \rightarrow 0 H=(1+\sqrt{2 / 3}) \sigma \approx 1.8 \sigma$, the pore still allows for a closed packed structure of two particle layers with 9 neighbors per particle, with $\mathrm{A}$ and $\mathrm{B}$ particles mixed. If the number of neighbors remains approximately constant, the reduction of available volume with the shrinkage of the pore width will induce demixing. The effect will still be present but less patent when going from $H \approx 3 \sigma$ to $H \approx 2 \sigma$. Large values of $\Delta$ will destroy this stabilizing effect, e.g., when $\Delta \approx 1 / 2$ volume exclusion will prevent the presence of unlike neighbors in adjacent layers. Small $\Delta$ values allow for this possibility and therefore, higher packing fractions of the stable mixture can be found, by which the non-monotonous dependence of the critical properties on the pore width is explained.

In summary, we have presented a detailed study of the effects of geometric confinement on symmetric mixtures of non-additive hard spheres. We have found that, as an overall trend, confinement tends to impede demixing, rising both critical densities and pressures, but interestingly for small degrees of non-additivity, a non-monotonous dependence is found. In fact, for certain values of the cross interaction, it is found that confinement can induce demixing by simple packing effects. In future works, we will address the effects of competition between energetic and steric contributions to the intermolecular potential and tunable wall interactions.

\section{ACKNOWLEDGMENTS}

The authors acknowledge the support from the Dirección General de Investigación Científica y Técnica under Grant Nos. FIS2010-15502 and FIS2013-47350-C5-4-R. The CSIC is also acknowledged for providing support in the form of the project PIE 201080E120.

${ }^{1}$ L. D. Gelb, K. Gubbins, R. Radhakrishnan, and M. Sliwinska-Bartkowiak, Rep. Prog. Phys. 62, 1573 (1999).

${ }^{2}$ J. Binney, N. Dowrick, A. Fisher, and M. Newman, The Theory of Critical Phenomena (Clarendon press, Oxford, 1993).

${ }^{3}$ K. Binder, Annu. Rev. Phys. Chem. 43, 33 (1992).

${ }^{4}$ Y. Rouault, J. Baschnagel, and K. Binder, J. Stat. Phys. 80, 1009 (1995).

${ }^{5}$ R. L. C. Vink, K. Binder, and J. Horbach, Phys. Rev. E 73, 056118 (2006).

${ }^{6}$ N. Severin, I. M. Sokolov, and J. P. Rabe, Langmuir 30, 3455 (2014).

${ }^{7}$ F. R. Hung, K. E. Gubbins, R. Radhakrishnan, K. Szostak, F. Béguin, G. Dudziak, and M. Sliwinska-Bartkowiak, Appl. Phys. Lett. 86, 103110 (2005).
${ }^{8}$ C. Alba-Simionesco, B. Coasne, G. Dosseh, G. Dudziak, K. E. G. R. Radhakrishnan, and M. Sliwinska-Bartkowiak, J. Phys.: Condens. Matter 18, R15 (2006).

${ }^{9}$ J. W. Biddle, V. Holten, and M. A. Anisimov, J. Chem. Phys. 141, 074504 (2014).

${ }^{10}$ S.-H. Chen, F. Mallamace, C.-Y. Mou, M. Broccio, C. Corsaro, A. Faraone, and L. Liu, Proc. Natl. Acad. Sci. U. S. A. 103, 12974-12978 (2006).

${ }^{11}$ C. E. Bertrand, Y. Zhang, and S.-H. Chen, Phys. Chem. Chem. Phys. 15, 721 (2012).

${ }^{12}$ R. L. C. Vink, A. D. Virgiliis, J. Horbach, and K. Binder, Phys. Rev. E 74, 031601 (2006).

${ }^{13}$ A. Fortini, M. Schmidt, and M. Dijkstra1, Phys. Rev. E 73, 051502 (2006).

${ }^{14}$ G. Pellicane, R. L. C. Vink, C. Caccamo, and H. Löwen, J. Phys.: Condens. Matter 20, 115101 (2008).

${ }^{15}$ J. Zausch, P. Virnau, K. Binder, J. Horbach, and R. L. Vink, J. Chem. Phys. 130, 064906 (2009)

${ }^{16}$ A. Fortini and M. Dijkstra, J. Phys.: Condens. Matter 18, L371 (2006).

${ }^{17}$ S. Asakura and F. Oosawa, J. Chem. Phys. 22, 1255 (1954).

${ }^{18}$ B. Widom and J. S. Rowlinson, J. Chem. Phys. 52, 1670 (1970).

${ }^{19}$ G. Johnson, H. Gould, J. Machta, and L. K. Chayes, Phys. Rev. Lett. 79, 2612 (1997).

${ }^{20}$ W. T. Góźdź, J. Chem. Phys. 122, 074505 (2005).

${ }^{21}$ F. Saija and P. V. Giaquinta, J. Chem. Phys. 117, 5780 (2002).

${ }^{22}$ E. Lomba, M. Alvarez, L. Lee, and N. G. Almarza, J. Chem. Phys. 104, 4180 (1996).

${ }^{23}$ W. T. Góźdź, J. Chem. Phys. 119, 3309 (2003).

${ }^{24}$ K. Jagannathan and A. Yethiraj, J. Chem. Phys. 118, 7907 (2003).

${ }^{25}$ A. Buhot, J. Chem. Phys. 122, 024105 (2005).

${ }^{26}$ Y. Duda, E. Vakarin, and J. Alejandre, J. Colloid Interface Sci. 258, 10 (2003).

${ }^{27}$ D. A. Kofke and E. D. Glandt, Mol. Phys. 64, 1105 (1988).

${ }^{28}$ D. Frenkel and B. Smit, Understanding Molecular Simulation (Academic, London, 2002).

${ }^{29}$ R. H. Swendsen and J.-S. Wang, Phys. Rev. Lett. 58, 86 (1987).

${ }^{30}$ U. Wolff, Phys. Rev. Lett. 62, 361 (1989).

${ }^{31}$ D. Stauffer and A. Aharony, Introduction to Percolation Theory, 2nd ed. (Taylor and Francis, London, 2003).

${ }^{32}$ E. de Miguel and G. Jackson, Mol. Phys. 104, 3717 (2006).

${ }^{33}$ E. de Miguel, N. G. Almarza, and G. Jackson, J. Chem. Phys. 127, 034707 (2007).

${ }^{34} \mathrm{~J}$. Henderson, "Statistical mechanical sum rules," in Fundamentals of Inhomogeneous Fluids (Marcel Dekker, New York, 1992), pp. 23-84.

${ }^{35}$ K. Binder, Z. Phys. B 43, 119 (1981).

${ }^{36}$ D. P. Landau and K. Binder, A Guide to Monte Carlo Simulations in Statistical Physics (Cambridge University, Cambridge, 2005).

${ }^{37}$ M. E. Fisher, Phys. Rev. 176, 257 (1968).

${ }^{38}$ N. G. Almarza, J. Tavares, and M. T. da Gama, Phys. Rev. E 85, 053102 (2012).

${ }^{39}$ L. G. López, D. H. Linares, and A. J. Ramirez-Pastor, Phys. Rev. E 85 , 053101 (2012).

${ }^{40}$ P. G. de Sanctis Lucentini and G. Pellicane, Phys. Rev. Lett. 101, 246101 (2008).

${ }^{41}$ J. Salas and A. K. Sokal, J. Stat. Phys. 98, 551 (2000).

${ }^{42}$ M. E. Fisher and H. Nakanishi, J. Chem. Phys. 75, 5857 (1981).

${ }^{43}$ R. Evans, J. Phys.: Condens. Matter 2, 8989 (1990). 